Crime, Histoire \& Sociétés / Crime, History \& Societies

Vol. 2, n² | 1998

Histoire de la criminalité et de la justice pénale en Amérique latine / Criminal justice history in Latin America

\title{
Médecine du crime, médecine de l'âme
}

\section{Michel Porret}

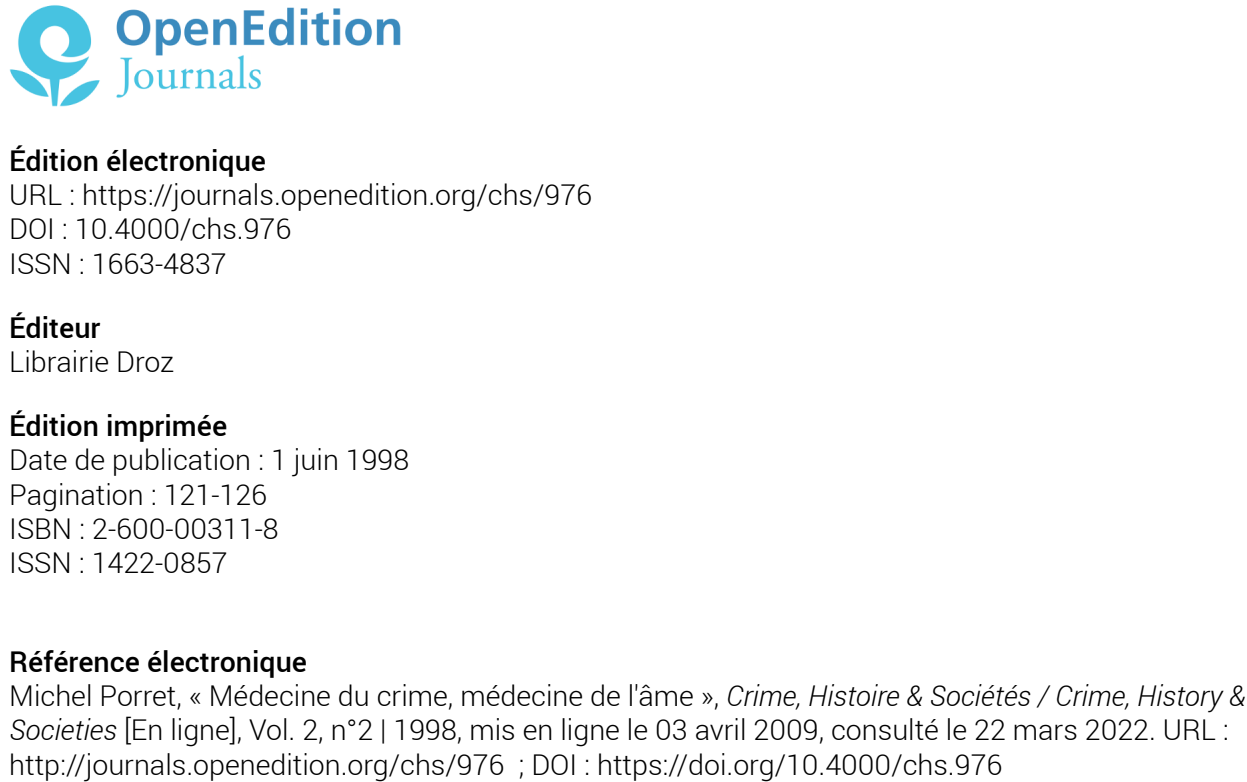

Édition électronique

URL : https://journals.openedition.org/chs/976

DOI : 10.4000/chs.976

ISSN : 1663-4837

Éditeur

Librairie Droz

Édition imprimée

Date de publication : 1 juin 1998

Pagination : 121-126

ISBN : 2-600-00311-8

ISSN : 1422-0857

Référence électronique

Michel Porret, " Médecine du crime, médecine de l'âme », Crime, Histoire \& Sociétés / Crime, History \&

Societies [En ligne], Vol. 2, n² | 1998, mis en ligne le 03 avril 2009, consulté le 22 mars 2022. URL

http://journals.openedition.org/chs/976 ; DOI : https://doi.org/10.4000/chs.976 


\section{Médecine du crime, médecine de l'âme}

Q enneville (Marc), Essai sur l'émergence d'un regard médical sur la crimi-

Rnalité en France (1785-1885). Thèse de doctorat d'Histoire nouveau régime, Université Paris 7 - Denis Diderot, Villeneuve d'Ascq, Presses universitaires du Septentrion (Thèse à la carte), 1997, 2 volumes, 920 p.;

Renneville (Marc), Drôle d'oiseau. Autobiographie d'un voyou à la Belle Époque, texte établi et présenté par Philippe Artières, Paris, Éditions Imago, 1998, $158 \mathrm{p}$.

$\mathrm{Au} \mathrm{XIX}^{\mathrm{e}}$ siècle, alors que le code pénal $(1791,1810)$ a mis fin à l'arbitraire de la justice criminelle de l'Ancien Régime et que la prison pénitentiaire, revers du contrat social, remplace l'«éclat des supplices" par la privation de liberté comme mode d'application des peines, entre psychiatrie, médecine légale et anthropologie criminelle, les territoires du judiciaire sont l'objet d'une qualification scientifique. Celle-ci traduit notamment l'idéal positiviste du temps et la médicalisation globale de l'anomie sociale qu'à la fin du siècle la sociologie durkheimienne cherche à modéliser. Fortement ancrée dans une culture de l'expertise judiciaire qui s'est affirmée dès la Renaissance au moins sous l'influence de la procédure inquisitoire, cette révolution positiviste dans la qualification des délits et dans la motivation des peines veut parfaire le vieil impératif de certitude recherché par les procureurs généraux ou les juges lorsqu'ils requièrent ou disent le droit au nom de l'État.

Visant à naturaliser et à «objectiver» toutes les « circonstances » du crime, cette alliance entre le pénal et la science est l'enjeu d'une médicalisation intense que la thèse de Marc Renneville, dans une approche d'histoire des sciences revendiquant les exigences socioculturelles d'un Jacques Roger, historicise en retournant aux sources médicales, médico-légales, psychiatriques, statistiques ou encore sociologiques du XIX ${ }^{\mathfrak{c}}$ siècle. Le siècle de Marx est en effet une époque où «toutes les formes de déviance $[. .$.$] ont subi une médicalisation partielle ou totale»: celle-ci$ cherche surtout à pronostiquer et à endiguer la récidive criminelle tout en humanisant l'application des peines. Alliant l'histoire sociale des transgressions à celle des idées sociales, pénale ou médicale ainsi que des doctrines anthropologiques, divisée en trois parties, quinze chapitres, une «Conclusion générale», à quoi s'ajoute une grosse bibliographie thématique des sources et des travaux secondaires, cette étude couvre une chronologique d'un siècle environ. En effet, Renneville, qui vient de consacrer un brillant article à l'épistémologie de Lombroso («Rationalité contextuelle et présupposé cognitif, le cas Lombroso ", Revue de synthèse, 1997, 118, $4^{\mathrm{e}} \mathrm{s}$., 4, p. 495-529), traque l'idéal de médicalisation du crime depuis l'«idéalisme des Lumières » (que l'on pourrait qualifier aussi d'utilitarisme pur et dur) jusqu'au 
moment final de l' «ancrage du regard médical sur la criminalité» (1848-1885). Si la France des idéologues (p. 135-152), des hygiénistes, des aliénistes et des criminologues contemporains de Lacassagne constitue la géographie intellectuelle de l'ouvrage (p. 521-774), la phrénologie de l'anglais Gall (1758-1828) et la criminologie de Lombroso (1835-1909), que Renneville inclut dans sa réflexion, montrent que la médicalisation du crime est une affaire européenne. Elle vise surtout à «gouverner la déviance " par l'incarcération et les enquêtes statistiques (p. 170-209). À la fin du siècle, ses retombées épistémologiques, via la «médicalisation de la folie » (p. 210-250), marquent les congrès de criminologie qui font de la récidive et de la «dangerosité» l'essentiel de leurs travaux.

Placé ici comme matrice épistémologique de la médicalisation du crime contre la tradition multiséculaire de qualification moralo-religieuse, 1 ' « idéalisme juridique des Lumières » est pourtant problématique dans l'analyse téléologique qu'en fait Renneville lorsqu'il pense la pratique pénale de l'Ancien Régime dans sa finalité révolutionnaire (légalité, codification, fixité des peines), creuset constructif d'une «nouvelle forme de gouvernementalité» (p. 50). On peine parfois à suivre le distinguo que Renneville établit entre « représentation» de la criminalité et doctrine encadrant les pratiques pénales (p. 37-95, passim): se fait-il historien de l'imaginaire social volontiers alarmiste envers l'insécurité ou historien des théories criminelles léguées par le jus romanum et le droit pénal de la monarchie? Au delà de la tentation holistique, peut-on vraiment ramener l'enracinement doctrinaire des pratiques pénales de l'Ancien Régime européen aux thèses singulières de l'Homme criminel (1644) du père oratorien Jean-François Sénault qui, parmi d'autres théologiens, lie le crime au péché ? Plus généralement, à la lumière de la pratique pénale révélée par les archives judiciaires et de nombreux travaux récents consacrés à celle-ci, il est difficile de montrer que la doctrine et la pratique criminelles, après l'Ordonnance de 1670 , soient inféodées à la théologie morale aussi fortement que le prétend l'auteur, même si certains doctrinaires (Muyart de Vouglans) favorables à un absolutisme de droit divin intransigeant flirtent avec un providentialisme judiciaire qui les pousse à récuser la réforme laïcisante des "philosophes». Pourtant, autant en France, que dans d'autres États européens comme Genève ou les Pays-Bas, la laïcisation du judiciaire progresse tranquillement au cours du XVIII siècle: le droit pénal révolutionnaire parachève un mouvement séculaire de rationalisation, initiée avec la fin de la grande chasse aux sorcières ( $c a$ 1650-1670) et exigée progressivement par maints magistrats du temps. Bien évidemment, la source des lois pénales, notamment dans les régimes monarchiques, repose dans la constitution juridique de la monarchie de droit divin: cet enracinement symbolique leur apporte une légitimité sacrale que le régicide récuse plutôt que d'instrumentaliser la « logique sacrificielle du bouc émissaire» (p. 83). De plus, il est ardu de penser la nature de la justice arbitraire de l'Ancien Régime à travers les sources qui en intentent le procès idéologique (réformateurs post-beccariens, intellectuels rousseaussistes de tous les bords, cahiers de doléances), à moins de vouloir faire l'histoire de ce réquisitoire politique que 1789 radicalise. Après les travaux de Bernard Schnapper, on sait bien, par exemple, que l'arbitraire, longtemps associé positivement au pouvoir de la médiation et de l'arbitrage des juges, devient une catégorie négative et polémique dès les années 1760 qui est utilisée pour incriminer les institutions «tyranniques» de l'absolutisme (roi, ministres, parlements, justice criminelle). Est-ce vraiment d'ailleurs le même réformisme anti-absolutiste qui agite dès les Lettres persanes un Montesquieu favorable 
à une monarchie tempérée par des corps intermédiaires (parlements), qui mobilise un Voltaire militant pour la tolérance religieuse lors de l'affaire Calas ou un Mercier qui vulgarise Rousseau en le radicalisant? Peut-on encore postuler que l' ' agitation philosophique et politique » de ces hommes de lettres prépare la révolution de 1789 ? Au contraire, après les écrits de Bronislaw Baczko ou de Roger Chartier, on sait que la culture politique de la Révolution française se légitime en plaçant son origine dans celle des Lumières. Le régicide est-il vraiment l'aboutissement du réformisme des philosophes? Incarnée par un Beccaria, la réforme pénale des Lumières, parfois ramenée à un catalogue simplificateur de ses auteurs (p. 57), à des généralités sur le jusnaturalisme (p. 85-95), ou à un résumé disproportionné de l'histoire politique du code pénal (p. 63-95), s'insère dans un horizon d'attentes judiciaires préparé et voulu par maints doctrinaires ou magistrats (juges, procureurs) de l'Ancien Régime. Théoricien du déclin comme signe de la continuité entre les civilisations, Volney est-il le doctrinaire le plus pertinent pour penser, autour de la figure de l' «homme sauvage» (métaphore du criminel?), la continuité et les ruptures pénales entre les Lumières et le $\mathrm{XIX}^{\mathrm{e}}$ siècle (p. 96-110)? Héritée de l'Ancien Régime (p. 112-134) et appuyée sur les travaux prophylactiques de la Société royale de médecine, la médicalisation progressive des institutions disciplinaires et hospitalières contribue certainement plus fortement à générer ce «bio pouvoir», matrice de la médecine du crime. Celui-ci, via les idéologues (Cabanis, Destutt de Tracy), les écoles étatiques de santé et de médecine ouvertes par la Révolution, induit la médecine légale scientifique (Fodéré, p. 162-169) et la criminologie, cette "police médicale » de la société qui trouve un relais mobilisateur dans les «préoccupations hygiénistes» des années 1795-1850 (p. 153-162).

Montrant finalement, à juste titre après Marcel Gauchet et Gladys Swain, que le modèle de la rupture foucaldienne entre l'Ancien Régime et le $\mathrm{XIX}^{\mathrm{e}}$ siècle est une fiction historiographique qui néglige l'idéal volontariste des Lumières en ce qui concerne l'institutionnalisation de la médecine, le discours criminologique et les pratiques pénales «nées » avec la modernité révolutionnaire, Renneville vise un but très ambitieux en voulant historiciser les discours scientifiques sur le passage à l'acte que formule l'anthropologie criminelle qui se médicalise jusqu'aux années 1890. Normalisant et élogieux pour le progrès scientifique, ces discours s'inscrivent dans une tradition morale (parfois religieuse) et judiciaire millénaire, laquelle naturalise progressivement la question criminelle. Si les pénalistes des Lumières, après Beccaria, accordent surtout une attention majeure aux conditions d'application des peines dont la dureté et l'archaïsme sont alors imputés à des régimes «despotiques», les médecins du crime au XIX ${ }^{e}$ siècle investissent l'âme des délinquants afin de le guérir et de prévenir la récidive perçue comme une pathologie morale que sonde l'anthropologie criminelle (p. 631-676). Celle-ci résulte des «manies» («monomanie», «lypémanie», «pyromanie», «kleptomanie», etc. p. 527-541) notamment homicides, dont l'étiologie, formulée par les aliénistes mais héritée de diverses doctrines des «passions de l'âme», permet de construire la thèse de la dégénérescence physique et morale des individus qualifiés de «crétins» ou de criminels (p. 542577); leurs vices se transmettent par atavisme familial et contamineraient la " partie saine de la société». Mis au service de la qualification judiciaire, ce vocabulaire médical devient l'instrument d'analyse de l'anomie criminelle: naturalisée, la transgression acquiert un statut pathologique que la science médicale diagnostique avec «certitude» (Morel, bientôt Lombroso) au nom d'un atavisme biologique que 
Lacassagne ou Tarde remplacent progressivement par un déterminisme social (p. 684-710) que marque tout de même l'évolutionnisme darwinien (p. 711-721). La thèse du criminel-malade (voire "criminel né »), puis du «criminel par profession », deviennent ainsi des topoi du discours scientifique dénonçant le «microbe moral» du crime. Ils induisent une représentation sociale et sécuritaire du délinquant que relaient après la Commune de 1871 l'imaginaire naturaliste et la presse populaire spécialisée dans le récit du fait divers criminel. L'imaginaire collectif s'enracine dans le positivisme scientifique qui construit les catégories de la «dangerosité » sociale dont le XIX ${ }^{\mathbf{e}}$ siècle se repaît jusqu'à la veille de la Grande guerre.

Confirmant finalement le diagnostic massif que les sciences humaines du XIX ${ }^{e}$ siècle portent sur l'anomie (atavique, le crime est une pathologie sociale, l'homo criminalis incarne un âme perverse qui est guérissable dans un corps délinquant), cet essai touffu doit se transformer en livre achevé à la hauteur de sa forte ambition. Il devra être expurgé des ruptures ou des articulations formelles d'une thèse de doctorat; il devra se concentrer plus systématiquement sur son objet (l'archéologie du savoir criminologique, sa diffusion, sa culture médicale) en hiérarchisant mieux les sources et les problématiques. L'ouvrage final devra renoncer aux trop nombreuses digressions parfois réductrices (absolutisme, Lumières, Bacon, matérialisme lockien, anthropologie des passions dès la Renaissance, Révolution de 1789, histoire politique, "pacte narratif» de l'historien, déterminisme causal, utopisme social, "psychiatrie scientifique», Kant, Nietzsche, etc.); il devra en outre briser avec la manie pesante de vouloir établir les «origines » et les généalogies du moindre objet étudié (cartésianisme, providentialisme et théorie de l'entendement chez Bossuet, idéologie, hygiénisme, médecine légale, Morel, etc.). Finalement, pour une lisibilité digne de cette originale recherche, l'ouvrage devrait être amputé de sa partie consacrée à l' " essor et déclin de la phrénologie (1808-1848)», car celle-ci est la matrice d'un livre en soi consacré à une sémiologie de la médecine clinique et à une symptomatologie morale de l'honnêteté et du vice (p. 252-418). Muni d'un index analytique et onomastique, le livre de Renneville affinera ainsi la connaissance de la médicalisation de l'anomie sociale et des thèses de la dégénérescence durant le second XIX siècle. Fondateur de la modernité judiciaire, cet aspect ressort bien de la meilleure partie de le recherche («Diffusion», p. 527-774) qui ouvre théoriquement sur la problématique de l'insertion de la doctrine médico-légale dans les pratiques de la répression pénale («Bilan, Perspectives», p. 792-805). Une bonne mesure des enjeux de cet apport scientifique sur la pratique judiciaire ne pourra ressortir d'une investigation massive sur les archives judiciaires du XIX ${ }^{e}$ siècle en traquant systématiquement la pratique de la médecine du crime. Comme le suggère sans cesse Renneville jusque dans ses conclusions, cette médicalisation vise un objectif réformiste inhérent à l'État de droit affirmé dès la Déclaration des droits de l'homme et du citoyen de 1789: la mise en place d'une «forme de contrôle social, plus douce et plus efficace parce que plus proche des individus » (p. 162) et dans lequel la guérison du criminel prime sur sa punition car il est devenu un sujet de droit en même temps qu'un objet de savoir pour la médecine du crime. Un tournant pourtant radical s'est amorcé durant ce long XIX ${ }^{\mathfrak{e}}$ siècle, c'est celui que montre Rennevile à toutes les pages de son livre: le fatalisme religieux a cédé le pas au fatalisme biologique; la laïcisation de la question criminelle culmine dans son approche médicale. Cette mutation dans la qualification de la criminalité induit de nouvelles pratiques pénales dont la prison devient le laboratoire social: la justice expiatoire de 
l'Ancien Régime, qui punissait dans son corps le criminel pécheur, est remplacée par une pénalité enracinée dans l'idéal thérapeuthique qui vise à guérir l'âme de l'homo criminalis décrit comme un dégénéré. Dès 1800 environ, la morale sociale a trouvé ainsi un point d'ancrage scientifique qui en fait une redoutable arme contre la marginalité ou la délinquance.

On mesure les enjeux et l'influence de la médecine du crime en lisant les «Souvenirs d'un moineau ou les Confidences d'un prisonnier » d'Emile Nouguier, jeune «apache», souteneur, chef d'une bande de voleurs, flirtant avec l'anarchisme et guillotiné en février 1890 pour meurtre à la prison de Saint-Paul (Lyon). Éditée par Philippe Artières (éditeur en 1995 des Mémoires du meurtrier Charle Double Charle Double. État psychologique et mental d'un inverti parricide, 1905, suivi de: Impressions d'un condamné Hermaphrodite mental!, Lille, Cahiers Gai-KitschCamp, 1995, $\mathrm{n}^{\circ}$ 5), cette autobiographie, qui aurait mérité une annotation moins minimaliste, résulte de la rencontre du meurtrier incarcéré et du fondateur de la criminologie française, Alexandre Lacassagne (1843-1924). Dans son laboratoire criminologique qu'est la prison de Saint-Paul, Lacassagne remarque que Nouguier rédige un journal dans lequel il note ce qui habite son esprit ou ce que suscitent ses lectures. Décidant d'exploiter les talents littéraires du jeune voyou repentant et mûr pour une introspection morale, Lacassagne, en grand confesseur/redresseur du crime, lui demande de rédiger une autobiographie. Aux yeux du médecin, ce texte doit constituer une «confession scientifique", utile pour connaître le milieu des apaches et les motivations intimes de l'homo criminalis qui peuvent ainsi être comparées aux aveux d'autres délinquants. Guidé et séduit par le médecin classificateur des déviances criminelles, qui d'ailleurs proposa à une dizaine d'autres prisonniers de rédiger de telles autobiographies, Nougier devient ainsi l'analyste de son propre milieu, ce que témoigne en outre son Dictionnaire d'argot, rédigé durant sa détention et réédité jusqu'à aujourd'hui (Clichy, 1987). Le texte illustre bien évidemment la grande précarité sociale du XIX ${ }^{\mathrm{e}}$ siècle et ses conséquences criminogènes; par déficit, il montre aussi l'idéal intégrateur de l'école obligatoire de la $\mathrm{III}^{\mathrm{e}}$ République; il éclaire en revers sombre la Belle Époque. Marquée par une culpabilité morale que l'on peut lire dans un langage empli de religiosité, cette confession est rédigée selon les règles d'une narration distanciée: Nouguier se confie à un moineau apprivoisé qui devient le greffier narrateur des confidences et de la déchéance sociale du jeune apache. La compagnie du volatile induit Nougier à recourir à un imaginaire ornithologique édifiant dont les métaphores ( «le nid » maternel quitté trop tôt, etc.) donnent sens à sa propre chute sociale.

Or au delà d'une sociologie de la marginalité, au-delà aussi des sources littéraires ou proverbiales de ce texte, c'est un peu l'idéal d'objectivation de la criminologie de la fin du XIX ${ }^{e}$ siècle que cette confession donne à lire. Écrire sa vie de criminel, c'est placer un parcours de vie négatif dans la finalité téléologique de la rédemption pénale, c'est avouer les «circonstances» qui conduisent au gibet, c'est donner sens, par un exercice raisonné d'écriture et de repentance, à des transgressions ou à des illégalismes (rébellion juvénile, errance, vol, violence, sexualité non conforme aux standards moraux, etc.) que la médecine du crime qualifie alors avec «certitude» de pathologie morale ou sociale. Entre péchés («Peut-être sommesnous venus au monde avec un péché originel sur notre conscience», p. 18) et atavisme moral («j'avais déjà en moi tous les germes du mal», p. 41), Nouguier intériorise par son écriture les catégories normatives que Lacassagne lui suggère en lui 
demandant de corriger et d'affiner ses confessions. Par exemple, il attribue son "penchant pour le vol » à sa désobéissance congénitale envers son père ou sa grandmère (p. 46) et aux «mauvais livres » (p. 50, 55-56) qui le détournent d'études studieuses. L'autobiographie signale ainsi la marginalisation croissante, subie puis choisie, que, dans un imaginaire peut-être rousseauisant, la mort de la mère (p. 38) et un déficit de moralité paternelle accentuent (le père de Nouguier vit en concubinage incestueux avec sa propre fille). Pour Lacassagne, le récit de vie devient ainsi un témoignage accablant sur un monde d'irrécupérables, il se transforme en document à charge qui nourrit, le cas échéant, les expertises et peut durcir l'accusation devant les Assises. En illustrant ce que la criminologie qualifie de «dangerosité sociale ", l' «autobiographie suscitée » de l'homme infâme légitime la répression pénale qui trouve dans les confidences arrachées une légitimité supplémentaire. Véritable «panoptique graphique » selon Artières, qui insiste dans sa postface sur la position commune qu'occupent un criminologue ou un anthropologue dans la constitution épistémologique de leurs sujets délinquants ou «indigènes», l'écriture ainsi contrainte par le questionnement du criminologue s'insère dans la construction «scientifique» de l'homo criminalis poussé à l'aveu introspectif.

Même à l'ère de la criminologie et de la médecine du crime triomphantes, cette technique réputée scientifique de la production de l'aveu prolonge pourtant la tradition du questionnement inquisitorial né vers la fin du XIV ${ }^{\mathrm{e}}$ siecle: aux preuves du crime reposant sur l'aveu, succèdent les preuves du bien-fondé de la criminologie. $\mathrm{Si}$, entre les quatre murs d'une cellule, l'aveu libère celui qui le rédige (Nouguier trouve un bonheur certain dans l'écriture de ses confidences que Lacassagne lui paie par quelques sous ou en améliorant son ordinaire de détenu), cette confession guidée par le criminologue finalise une forme moderne de providentialisme expiatoire liant la déchéance sociale, illustrée par l'autobiographie, au châtiment suprême : «Je maudis ma destinée quand je vois ce que j'aurais pu être et ce que je suis. Pour moi, vois-tu, je ne puis m'empêcher de dire en terminant: c'était écrit, ce qui est, ce qui doit être, sera » (p. 138). Si avant de monter sur le gibet Nouguier a voulu parler de «lui-même», donner sens à sa vie à travers sa propre subjectivité stigmatisante, ce projet échoue, car il est vite rattrapé par les exigences normatives de Lacassagne qui transforme le jeune apache en objet de connaissance pour la criminologie au moment où elle offre son savoir à la justice dans la guerre que la société d'alors mène contre les marginaux de tous bords. Cette autobiographie suscitée illustre ce que l'anthropologie criminelle d'alors ne pouvait admettre: la connaissance «objective » de l'homo criminalis est illusoire car elle repose en fait sur un modèle moral, préalablement construit, d'être humain corrompu. Sans le savoir, Nouguier conforte cette idéologie moralisante que son mentor Lacassagne lui inculque en lui arrachant les huit confidences d'un parcours de vie au déroulement inexorable: "C'était la route du crime, la route de la prison, du bagne et de l'échafaud» (p. 44). Naturaliste et moralisante, la médecine du crime reste une médecine de l'âme.

Michel Porret

Université de Genève

Département d'histoire générale

Unité d'histoire moderne

5 , rue Saint-Ours

CH-1211 Genève 5

Michel.Porret@lettres.unige.ch 